\title{
Characterisation of Yersinia Secretion Apparatus - Pathogenicity Island (Ysa-PI) of Yersinia enterocolitica 1B/O8 in Poland: an Idle Ysa is a Specific Hallmark of the Epidemic Sensu Stricto Strain
}

\author{
TOMASZ WOŁKOWICZ*, KATARZYNA ZACHARCZUK, NATALIA ROKOSZ-CHUDZIAK, \\ WALDEMAR RASTAWICKI and RAFAŁ GIERCZYŃSKI
}

National Institute of Public Health - National Institute of Hygiene, Warsaw, Poland

Submitted 12 June 2014, revised 24 February 2015, accepted 10 March 2015

Abstract

Yersinia secretion apparatus (Ysa), the chromosomal type three secretion system (T3SS) is considered to contribute to virulence of highpathogenicity Yersina enterocolitica biovar 1B. DNA-sequence of Ysa pathogenicity island was determined for clinical isolate DM0110 of Y. enterocolitica 1B/O8 with origin in Poland. We found a premature stop-codon in the regulatory gene ysrR (mutation at position 269). Altered $y s r R$ was detected in all tested 78 isolates of Y. enterocolitica 1B/O8 collected from clinical samples in Poland from 2004 to 2013. Since aberrations in YsrR are considered to inactivate Ysa, our findings may suggest Ysa is not indispensable for Y. enterocolitica 1B/O8 to infect humans.

Key words: Y.enterocolitica 1B/O8, T3SS, virulence, Ysa, Ysa-PI

Yersina enterocolitica is a causative agent of gastrointestinal disorders in humans with a variety of clinical manifestations, including reactive arthritis and erythema nodosum (Bottone, 1997). Pathogenic strains of Y. enterocolitica harbour a suit of virulence factors encoded on virulence plasmid pYV and the chromosome. Strains of bioserotype 1B/O8 are considered lethal to mouse and highly pathogenic to humans (Aulisio et al., 1983). Y. enterocolitica 1B/O8 was originally reported in northern America in the 70s and 80 s of the past century. In the early 90 s bioserotype 1B/O8 disappeared in America and emerged in Japan (Ichinohe et al., 1991). The first case of human Y. enterocolitica 1B/O8 infection in Europe was reported in Germany (Schubert et al., 2003). However, this bacterium is nowadays most numerously isolated in Poland, where 224 human clinical isolates were collected from 2004 to 2013. Y. enterocolitica 1B/O8 isolates collected were tightly clonal (Gierczyński etal., 2009; Zacharczuk, 2012). The bacterium is an important causative agent of yersiniosis in Poland as shown by serological studies (Rastawicki et al., 2013).

High pathogenicity of Y.enterocolitica $1 \mathrm{~B} / \mathrm{O} 8$ is attributed to virulence factors encoded in the chromosome. Chromosomal type III protein secretion system (T3SS) named Yersinia secretion apparatus (Ysa) is con- sidered a potential virulence factor of the bacterium. Ysa was found to have specific induction conditions - nutrient rich medium supplemented with a high salt concentration $(190-400 \mathrm{mM} \mathrm{NaCl}$ or $\mathrm{KCl})$ at a moderate temperature $\left(26-28^{\circ} \mathrm{C}\right.$ ) (Haller et al., 2000, Venecia and Young, 2005). Therefore, Ysa activity was initially considered as limited to the gastrointestinal tissues. The T3SS was shown to translocate some specific proteins named Yersinia secreted proteins (Ysps) (Haller et al., 2000, Venecia and Young 2005). Moreover, Ysa was found to secrete some of pYV-encoded Yersinia outer proteins (Yops) (Venecia and Young 2005). In recent studies, Ysa was proposed to act also at the systemic phase of the disease (Bent et al., 2013). All the aforementioned findings were derived from investigations conducted on laboratory reference strainsof Y. enterocolitica $1 \mathrm{~B} / \mathrm{O} 8$ and their mutants. However, little is known about Ysa activity in clinical isolates. Therefore we decided to investigate the Ysa activity in clinical isolates of Y. enterocolitica 1B/O8 collected in Poland.

Clinical strain DM0110 of Y. enterocolitica 1B/O8 originated in Poland in 2005 and was selected for DNAsequencing of the complete Ysa pathogenicity-island (Ysa-PI). DNA-sequencing was conducted by Sanger method using a BigDye Terminator v3.1 (Applied Biosystem). A library of 1.5-2.5 kbp fragments of Ysa-PI

\footnotetext{
* Corresponding author: T. Wołkowicz, National Institute of Public Health - National Institute of Hygiene, Warsaw, Poland; e-mail: twolkowicz@pzh.gov.pl
} 
Table I

Y. enterocolitica 1B/O8 reference strains and clinical isolates tested in this study

\begin{tabular}{|c|c|c|c|c|}
\hline $\begin{array}{c}\text { Strain ID } \\
\text { (no. isolates) }\end{array}$ & Source $^{a}$ & $\begin{array}{c}\text { Year } \\
\text { of isolation }\end{array}$ & $\begin{array}{c}\text { Ysps } \\
\text { secretion }^{\mathrm{b}}\end{array}$ & $y s r R$ type $^{c}$ \\
\hline WA-314 & Laboratory, MPI & NA & + & Wild \\
\hline 1105 & Laboratory, IP & NA & + & Wild \\
\hline 13804 & Laboratory, IP & NA & + & Wild \\
\hline 17451 & Laboratory, IP & NA & + & Wild \\
\hline 20167 & Laboratory, IP & NA & + & Wild \\
\hline 20169 & Laboratory, IP & NA & + & Wild \\
\hline 20175 & Laboratory, IP & NA & + & Wild \\
\hline 20176 & Laboratory, IP & NA & + & Wild \\
\hline 20178 & Laboratory, IP & NA & + & Wild \\
\hline 20189 & Laboratory, IP & NA & + & Wild \\
\hline 20232 & Laboratory, IP & NA & + & Wild \\
\hline DM0099 & Clinical sample & 2008 & - & Altered \\
\hline DM0102 & Clinical sample & 2008 & - & Altered \\
\hline DM0110 & Clinical sample & 2005 & - & Altered \\
\hline DM0147 & Clinical sample & 2006 & - & Altered \\
\hline DM0149 & Clinical sample & 2005 & - & Altered \\
\hline DM0150 & Clinical sample & 2004 & - & Altered \\
\hline DM0209 & Clinical sample & 2009 & - & Altered \\
\hline DM0249 & Clinical sample & 2009 & - & Altered \\
\hline$(\mathrm{n}=70)$ & Clinical samples & 2004-2013 & Not tested & Altered \\
\hline
\end{tabular}

a MPI (Max Von Pettenkofer Institut, Munich, Germany); IP (Pasteur Institute, Paris, France); b (+) for Ysps secretion (-) for no Ysps secretion; c result of PCR-RFLP assay for position 269 in the $y s r R$ gene; NA - not applicable

of DM0110 was cloned in plasmid pJet1.2/blunt Cloning Vector (Thermo Scientific, Lithuania) prior to DNA sequencing. Sequencing results were read on automated capillary sequencers in a biotechnological company (Genomed, Poland). Reviewed complete 30379bp - long YSA-PI cluster was deposited in GenBank (www. ncbi.nlm.nih.gov) database under no. KC784374.1.

Further examinations were performed on a number of 78 clinical isolates and 11 reference strains (Table I). PFGE typing (data not shown) showed that all the isolates were tightly-clonal and belonged to the previously described XbaI genotype II (Gierczyński et al., 2009). Ysa and Ysps proteins were visualised by SDS-PAGE assay. The assay was performed in accordance with the procedure described by Matsumoto and Young (2006). Eight Y. enterocolitica 1B/O8 clinical isolates originating in Poland were tested by SDS-PAGE together with DM0110 and the 11 reference strains listed in Table I. Bands specific for Ysps and Ysa proteins were detected for the reference strains only. In contrast to the reference strain WA-314 no Ysps could be detected for clinical isolate DM0110 in duplicate experiments (Fig. 1A). Ysps were also lacking in seven randomly selected clinical isolates (Fig. 1B).

The Ysa-PI sequence of DM0110 was aligned to the homologous loci deposited in commonly acces- sible databases using CLC Sequence Viewer 7 (CLC Inc, Denmark). Comparison of nucleotide and deduced amino-acid sequences of Ysa-PI of Y.enterocolitica 1B/O8 reference strains: 8081 (NC_008800.1), A127/90 (AF369954.1 and AY100449.2) and WA314 (AKKR01000083) was conducted to trace amino-acid substitutions and other alterations.

DM0110 revealed 99\% homology to Ysa-PI of reference strains 8081, A127/90 and WA314. No structural aberrations were observed. The only significant mutation specific for tested DM0110 was found in $y s r R$ gene that is part of the YsrRST phosphorelay system (Venecia and Young 2005, Walker et al., 2010). Single nucleotide substitution (C to A) at position 269 of $y s r R$ resulted in a premature stop-codon that terminates translation of YsrR at position 90. No such mutation was found in the reference Y.enterocolitica 1B/O 8 strains examined in this study.

To verify whether the stop-codon prematurely terminates the expression of $y$ srRin DM0110, the complete $y s r R$ gene (711 bp length) from DM0110 was cloned to expressing vector and examined in Escherichia coli. Expression system pET-30 Ek/LIC Vector Kit (Novagen, USA) was used according to the manufacturer's instruction. The unaltered $y s r R$ gene from strain WA-314 was used as a reference. Recombinant proteins of both 


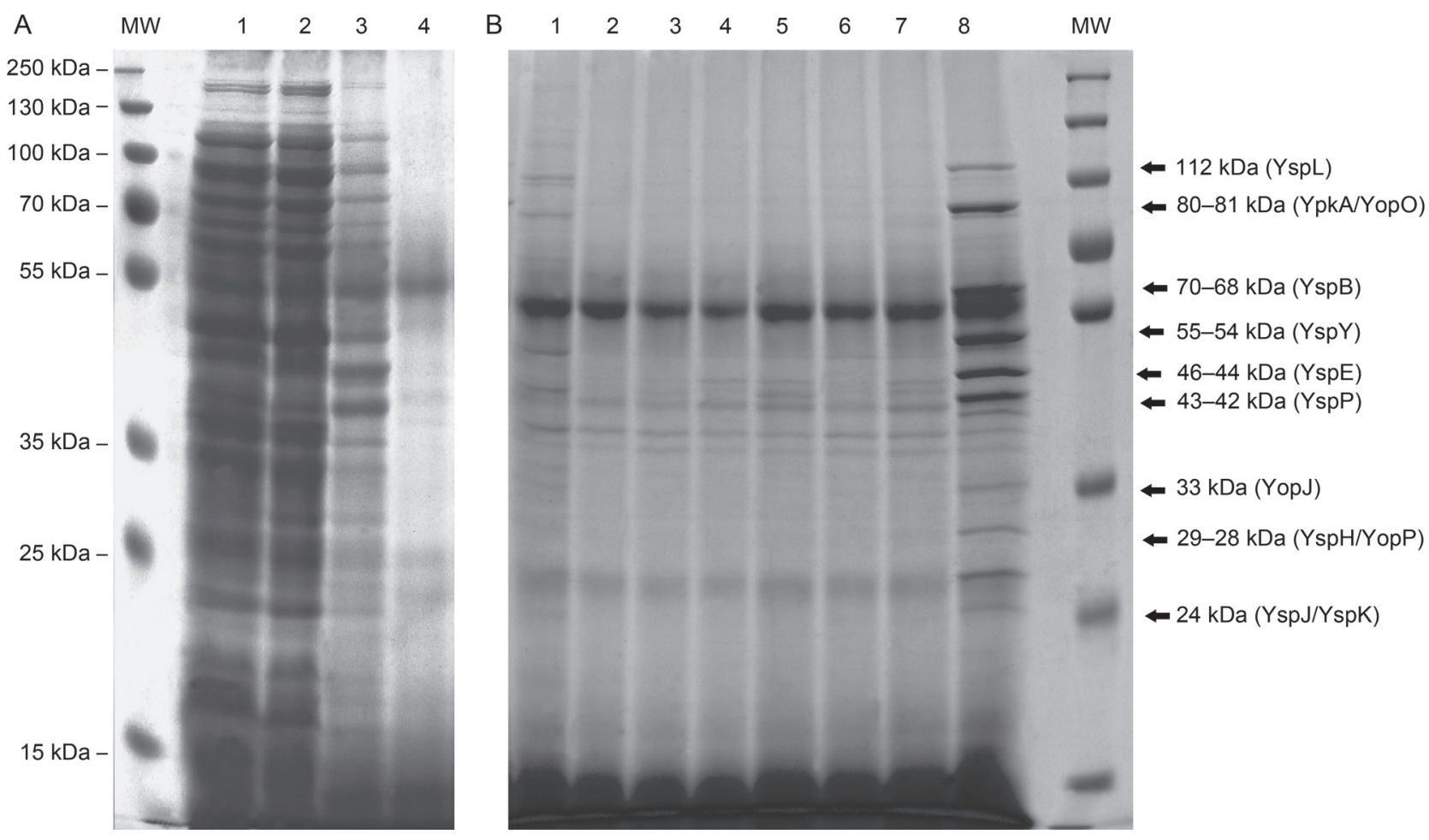

Fig. 1. SDS-PAGE profiles of secreted proteins Ysa-Ysp of Y. enterocolitica 1B/O8 reference strains and clinical isolates from Poland A: lines 1 and 2 - strain WA-314; 3 - strain 20175; 4 - clinical isolate DM0110. B: lines 1 to 7 - clinical isolates: DM0150, DM0147, DM0099, DM0102, DM0149, DM0249, DM0209 respectively; line 8 - strain WA-314; MW - molecular weight standard

DM0110 and WA-314 were purified on column with $\mathrm{Ni}^{2+}$-ISA His-Binding Resin (Novagen, USA). Molecular weight of the expressed recombinant proteins was determined by SDS-PAGE according to Laemmli (1970).The recombinant YsrR protein of DM0110 was about $10 \mathrm{kDa}$, while the YsrR recombinant of the unaltered $y s r R$ gene from WA- 314 was $27 \mathrm{kDa}$. These results confirmed that the premature stop-codon in $y s r R$ is active in vitro. Further analysis in silico performed with Domain Enhanced Lookup Time Accelerated BLAST (DELTA-BLAST) algorithm showed that YsrR protein REC domain is only half-expressed in DM0110 while the LuxR-C-like domain is lacking.

To determine whether other clinical isolates of Y. enterocolitica 1B/O8 in Poland carry unaltered or prematurely-terminated $y s r R$, PCR-RFLP assay targeting the unaltered (wild-type) ysrR gene was developed. Primers (ysrR-F 5'ATGACACAAACGAAAACGCTCAAT and ysrR-R 5'TTATAGAGAAATTTCATGAGCAT) were used to amplify the $711 \mathrm{bp}$ PCR-product of the $y s r R$ gene. PCR was conducted as described previously (Wołkowicz et al., 2014). The $y s r R$ PCR-product was further digested by BtsI endonuclease (New England BioLabs) as recommended by the manufacturer. The PCR-product of the unaltered, wild-type $y$ srR gene yielded two fragments of expected size 268 and $445 \mathrm{bp}$. In the case of mutation in position 269 of $y s r R$, the PCR-product remained undigested. In each PCRRFLP experiment the reference strain WA-314 was used as a control for the wild-type $y s r R$. DM0110 and all the tested clinical isolates listed in Table I were shown by the PCR-RFLP assay to bear $y$ srR gene altered in position specific for the premature stop-codon. No alterations were detected in ten reference strains of Y. enterocolitica 1B/O8 (Table I) previously found to secrete Ysps (Rokosz-Chudziak et al., 2013).

In the past two decades Y.enterocolitica 1B/O8 emerged in Japan, Germany and Poland. Since 2004 this bacterium has been isolated in Poland from humans with clinically confirmed yersiniosis. Noteworthy, all clinical isolates of Y.enterocolitica 1B/O8 in Poland were found to be tightly clonal (Gierczyński et al., 2009, Zacharczuk, 2013). Y. enterocolitica 1B/O8 was isolated from a variety of clinical samples including stool and blood. We, therefore, assumed our collections of clinical isolates constitute an excellent material to study Ysa activity in human yersiniosis.

The results obtained in this study are, however, surprising when compared with other reports on Ysa T3SS published to date (Venecia and Young 2005, Matsumoto and Young 2006, Mildiner-Earley et al., 2007, Bent et al., 2013). First of all we showed that clinical isolates of Y.enterocolitica 1B/O8 collected in Poland from 2004 to 2009 were unable to secrete Ysps. Moreover, clinical isolate DM0110 was unable to produce Ysps under a variety of inducible conditions described elsewhere (Mildiner-Earley et al., 2007, Witkowski et al., 2008) (data not shown). These findings together have 
prompted us to search Ysa-PI of DM0110 for possible reasons for Ysa inactivation. One particularly interesting mutation in the $y s r R$ gene of YsrRST phosphorelay system was detected. This mutation triggers the premature stop-codon that early terminates the translation of regulatory gene $y s r R$.

The YsrRST phosphorelay system is considered the major regulatory system for YSA-PI (Venecia and Young, 2005, Walker et al., 2010). Consequently, the premature stop-codon in $y s r R$ may play a key role in Ysa T3SS silencing in Y. enterocolitica 1B/O8 circulating in Poland. Noteworthy, disruption of the $y s r R$ gene has been already reported to inactivate Ysps secretion by Ysa T3SS. Similarly to strain DM0110, a ysrR mutant of Y. enterocolitica 1B/O8 strain JB580v, constructed by Venecia and Young (2005), was also found unable to secrete Ysps.

Our results showed that expression of $y s r R$ in the clinical strain of Y. enterocolitica 1B/O8 is terminated at one third of the functional protein. Important REC and LuxR-C-like domains of the functional YsrR are, therefore, lacking or partially expressed in Y. enterocolitica 1B/O8 circulating in Poland. Interestingly, PCRRFLP test showed that all investigated clinical isolates from Poland bear the altered $y s r R$ gene. This finding corresponds with the strong clonality of clinical isolates of Y. enterocolitica 1B/O8 in Poland revealed by PFGE. Therefore, we conclude that the premature stop-codon in the $y s r R$ gene may serve as a hallmark of the epidemic sensu stricto strain of $Y$. enterocolitica 1B/O8 in Poland.

To the best of our knowledge, dysfunctional YSA$\mathrm{PI}$ has not been yet reported in Y. enterocolitica 1B/O8 from patients with clinically confirmed yersiniosis. Our findings together may, however, suggest that Ysa T3SS is not indispensable for Y. enterocolitica 1B/O8 to cause infection in human.

Preliminary results of this study were, in part, presented during the $11^{\text {th }}$ International Symposium on Yersinia, Suzhou, China, 24-28 June 2013 (Rastawicki W., Szych J., Rokosz N., Zacharczuk K., Wołkowicz T. and Gierczyński R. The emergence of high-pathogenicity Yersinia enterocolitica bioserotype 1B/O8 infections in Poland. Final Program Yersinia 11 ${ }^{\text {th }}$, pp. 74).

\section{Acknowledgements}

This study was supported by grant NN 401076039 from National Science Centre of Poland. We are grateful to dr Elisabeth Carniel from Pasteur Institute, Paris, France and dr Alexander Rakin from The Max von Pettenkofer-Institute of Hygiene and Medical Microbiology, Munich, Germany for support in the reference strains of $Y$. enterocolitica $1 \mathrm{~B} / \mathrm{O} 8$.

\section{Literature}

Aulisio C.C., W.E. Hill, J.T. Stanfield and R.L. Sellers Jr. 1983. Evaluation of virulence factor testing and characteristics of pathogenicity in Yersinia enterocolitica. Infect. Immun. 40: 330-335.
Bent Z.W., S.S. Branda and G.M. Young. 2013. The Yersinia enterocolitica Ysa type III secretion system is expressed during infections both in vitro and in vivo. Microbiology open 2: 962-975.

Bottone E.J. 1997. Yersinia enterocolitica: the charisma continues. Clin. Microbiol. Rev. 10: 257-276.

Chatterjee R., P.K. Halder and S. Datt. 2013. Identification and molecular characterisation of YsaL (YE3555): a novel negative regulator of YsaN ATPase in type three secretion system of enteropathogenic bacteria Yersinia enterocolitica. PLoS One. 8: e75028.

Garzetti D., H. Bouabe, J. Heesemann and A. Rakin. 2012. Tracing genomic variations in two highly virulent Yersinia enterocolitica strains with unequal ability to compete for host colonization. BMC Genomics 13: 467.

Gierczyński R., J. Szych, W. Rastawicki, S. Wardak and M. Jagielski. 2009. Molecular characterization of human clinical isolates of Yersinia enterocolitica bioserotype 1B/O8 in Poland: emergence and dissemination of three highly related clones. J. Clin. Micobiol. 47: $1225-1228$.

Haller J.C., S. Carlson, K.J. Pederson and D.E. Pierson. 2000. A chromosomally encoded type III secretion pathway in Yersinia enterocolitica is important in virulence. Mol. Microbiol. 36: 1436-1446. Ichinohe H., M. Yoshioka, H. Fukushima, S. Kaneko and T. Maruyama. 1991. First isolation of Yersinia enterocolitica serotype O:8 in Japan. J. Clin. Microbiol. 29: 846-847.

Laemmli U.K. 1970. Cleavage of structural proteins during the assembly of the head ofbacteriophage T4. Nature 227: 680-685.

Matsumoto H. and G.M. Young. 2006. Proteomic and functional analysis of the suite of Ysp proteins exported by the Ysa type III secretion system of Yersinia enterocolitica biovar 1B. Mol. Microbiol. 59: 689-706.

Mildiner-Earley S., K.A. Walker and V.L. Miller. 2007. Environmental stimuli expression of the Ysa type three secretion locus. In: Perry R.D. and J.D. Fetherston (eds.), pp. 211-213. The genus Yersinia: from genomics to function. Springer Science-Business Media, LLC., USA.

Rastawicki W., J. Szych, N. Rokosz, K. Zacharczuk and R. Gierczyński. 2013. Seasonality of Yersinia enterocolitica bioserotype 1B/O:8 infections in Poland. Epidemiol. Infect. 141: 2039-2042.

Rokosz-Chudziak N., W. Rastawicki, K. Zacharczuk and R. Gierczyński. 2013. Electrophoretic and immunological analysis of native proteins secreted in vitro under conditions inducing Ysa (Yersinia secretion apparatus) by clinical isolates of Yersinia enterocolitica 1B/O8 in Poland (in Polish). Med. Dośw. Mikrobiol. 65: 245-254.

Schubert S., J. Bockemühl, U. Brendler and J. Heesemann. 2003. First isolation of virulent Yersinia enterocolitica O8, biotype 1B in Germany. Eur. J. Clin. Microbiol. Infect. Dis. 22: 66-68.

Venecia K. and G.M. Young. 2005. Environmental regulation and virulence attributes of the Ysa type III secretion system of Yersinia enterocolitica biovar 1B. Infect. Immun. 73: 5961-5977.

Walker K.A., M.W. Obrist, S. Mildiner-Earley and V.L. Miller. 2010. Identification of YsrT and evidence that YsrRST constitute a unique phosphorelay system in Yersinia enterocolitica. J. Bacteriol. 192: 5887-5897.

Witkowski S.E., K.A. Walker and V.L. Miller. 2008. YspM, a newly identified Ysa tyle III secreted protein of Yersinia enterocolitica. J. Bacteriol. 190: 7315-7325.

Wołkowicz T., N. Wolaniuk, K. Zacharczuk, R. Gierczyński, N. Rokosz and W. Rastawicki. 2014. Development of molecular PCR-RFLP test for identification of the epidemic strain of Y. enterocolitica bioserotype 1B/O8 circulating in Poland since 2004 (in Polish). Med. Dośw. Mikrobiol. 66: 89-98.

Zacharczuk K. 2012. Ph.D. Thesis, Molecular characterisation of human clinical isolates of Yersinia enterocolitica 1B/O8 collected in Poland in 2009. National Institute of Public Health-National Institute of Hygiene. Warsaw, Poland. 